\title{
La lengua del otro: política y erótica de la traducción
}

\author{
Ángeles Carreres \\ University of Cambridge
}

\begin{abstract}
Si j’avais à risquer, Dieu m'en garde, une seule définition de la déconstruction, brève, elliptique, économique comme un mot d'ordre, je dirais sans phrase: plus d'une langue. (Derrida, Mémoires 38)

Bref: comment ne pas parler de soi? Mais aussi bien: comment le faire sans se laisser inventer par l'autre? ou sans inventer l'autre? (Derrida, Psyché 562)

Accompagnons ce mouvement d'amour, le geste de cet aimant (liebend) qui oeuvre dans la traduction. Il ne reproduit pas, ne restitue pas, ne représente pas, pour l'essentiel il ne rend pas le sens de l'original, sauf en ce point de contact ou de caresse, l'infiniment petit du sens. (Derrida, Psyché 223)
\end{abstract}

El auge actual de los estudios culturales coincide con un renovado interés en la traducción. El trasvase entre lenguas se nos presenta como un lugar propicio desde donde aproximarnos a los mecanismos de la relación con el otro (el otro lingüístico, cultural, etc.) y, más profundamente, con la otredad dentro de lo que creíamos propio. Tras el llamado "giro ético" en el pensamiento de Derrida, la presencia de la traducción se hace aún más notoria en sus textos. Pero más allá de las referencias explícitas que encontramos, lo realmente interesante es observar cómo el proceso de traducción—con los problemas de reproductibilidad, endeudamiento, suplementariedad, etc. que conlleva - posee una estrecha afinidad con la estrategia deconstructiva. Si hablando de deconstrucción es posible aplicar aún la noción de paradigma, podría decirse que en el paradigma de la traducción encuentra el proceder deconstructivo su reflejo especular. Como ha señalado Barbara Johnson, es precisamente la imposibilidad de sentirse en casa en la propia lengua materna la que constituye el núcleo de la filosofía de Derrida. En el acto de traducción, la confrontación con la lengua del otro pone de manifiesto ese extrañamiento de nosotros mismos al que también alude Paul de Man en su ensayo sobre Die Aufgabe des Übersetzers:

We think we are at ease in our own language, we feel a coziness, a familiarity, a shelter in the language we call our own, in which we think that we are not alienated. What the translation reveals is that this alienation is at its strongest in our relation to our own original language, that the original language within which we are engaged is disarticulated in a way which imposes upon us a particular alienation, a particular suffering. (84)

Cada lengua de la que se traduce o a la que se traduce es ya en sí misma un sistema profundamente marcado por la traducción. Al referirnos comúnmente a tal o cual lengua como una unidad actúa ya un proceso de abstracción que encubre y silencia el entramado de superposiciones, intersecciones y contaminaciones que la integran. No existe pues una lengua de pureza intacta, sino diversas concreciones de una red plurilingüe. A nivel textual, ese "estar entre lenguas" (Zwischensprachlichkeit) resulta en la imposibilidad de un discurso netamente monolingüe, tema que Derrida aborda en Le monolinguisme de l'autre ou la prothèse de l'origine. 
En el capítulo inicial, Derrida nos pide que imaginemos una situación en la que un individuo francófono integrado en la cultura francesa nos dijese:

\begin{abstract}
"Je n'ai qu'une langue, ce n" est pas la mienne. . . Je suis monolingue. . . Or jamais cette langue, la seule que je sois ainsi voué à parler, tant que parler me sera possible, à la vie à la mort, cette seule langue, vois-tu, jamais ce ne sera la mienne. Jamais elle ne le fut en vérité." (Le monoliguisme 13-14)
\end{abstract}

Esta aseveración nos parecería una contradicción pragmática, en tanto que estaríamos viendo realizada de hecho ante nosotros la misma cosa que se nos está negando. Sin salir de esta retórica de la paradoja, Derrida enuncia dos postulados que constituyen para él la" "ley" de la traducción, o mejor dicho, la traducción como ley:

Car cette double postulation,

-On ne parle jamais qu'une seule langue...

(oui mais)

-On ne parle jamais une seule langue...

ce n'est pas seulement la loi même de ce qu'on appelle la traduction. Ce serait la loi ellemême comme traduction. (Le monolinguisme 25)

En efecto, la lengua que hablamos, nuestro idioma personal, es sólo uno: el nuestro. Sin embargo, éste se encuentra penetrado en todas las direcciones por implantes (greffes) procedentes de otras lenguas, de otros idiomas, de modo que la empresa de encontrar la propia identidad lingüística se convierte en un perpetuo no encontrarse. Como apunta Derrida, es este plurilingüismo esencial el que impone la necesidad y la imposibilidad de la traducción, su ley. ${ }^{1}$ Así, la contradicción de que sólo hablamos una lengua que ni es una ni es nuestra lleva a David Wills en su respuesta a Derrida a concluir que "siempre que hablamos, traducimos" (51).

Lo primero que nos sorprende al comenzar a leer Le monolinguisme de l'autre es su tono de implicación personal, nada frecuente en los textos de Derrida. La cuestión de la identificación cultural y lingüística afecta directamente al autor en razón de su origen francomagrebí. Jacques Derrida, junto con más de cien mil judíos argelinos, fue privado de la nacionalidad francesa en la época de la ocupación alemana. El estado que en 1870 por el decreto Crémieux había otorgado la nacionalidad francesa a los judíos argelinos respaldando así su integración cultural se la quita medio siglo más tarde. A Derrida como a tantos otros no se les dio opción: les fue negado el contacto estrecho con la lengua árabe o berebere, haciendo del idioma del vecino más próximo el más ajeno, mientras que la lengua que se les otorgó/impuso—el francés—funcionaba como instrumento de colonización y no ofrecía unas mínimas garantías de identificación cultural estable.

En Derrida encontramos un buen ejemplo de una relación amor-odio con su lengua fruto de una identificación sentida como precaria y artificial: en L'oreille de l'autre afirma escribir contra la lengua francesa, esa lengua de la metrópoli que es y no es la suya (148). Por otro lado, Derrida evoca la enorme fascinación que le produjo el descubrimiento de la lite-

\footnotetext{
${ }^{1}$ También en "Living On: Border Lines" encontramos una afirmación paralela: "One never writes either in one's language or in a foreign language" (101).
} 
ratura y la fílosofía francesas. Aún más, admite - con cierto sentimiento de culpa sentir un rechazo instintivo por las hablas del francés que discrepen de la norma parisina y confiesa su esfuerzo por borrar de su propia dicción cualquier rasgo dialectal que pueda dejar entrever su origen argelino. Este dato significativo es revelador del grado de autonegación que el imperialismo lingüístico puede llegar a causar. Como apunta Johnson, es justamente a través de la lengua extranjera que volvemos a enfrentarnos a esa intimidad hecha de amor y odio hacia nuestra propia lengua materna.

El enfoque distinto de Le monolinguisme de l'autre, más cercano a la experiencia personal de su autor, aporta nuevos matices a cuestiones clave tratadas ya en obras anteriores, entre ellas la del texto original. La lengua que se ha hablado y escuchado desde la cuna no puede llamarse propia, es siempre algo prestado, algo que viene del otro. ${ }^{2}$ Es, en definitiva, una lengua de traducción, pero una traducción sin lengua de partida. No existe un original al cual remitirla, ni siquiera una instancia supletoria que pueda cumplir esa función tranquilizadora. Este suplemento sólo se encuentra presente en su ausencia, como deseo no cumplido de un objeto imposible: el deseo de una lengua primigenia que pueda llamarse propia. El punto de referencia al que el yo tiene que remitirse para configurarse no existe, no ha existido nunca. La única verdad posible para el yo tiene que ser inventada, producida. Pero, ¿no es siempre la verdad cuestión de producción? ${ }^{3}$ En este sentido, es posible interpretar la falta de verdades absolutas que cierren y aseguren el discurso bajo el modelo aquí aplicado a la traducción: cualquier discurso puede ser entendido en su precariedad consustancial como una traducción cuyo texto original, cuya versión plena, no es más que un deseo imposible. Cada texto-siempre segundo-busca así escribirse sobre las líneas borradas de un texto primero que el deseo trata en vano de reinventar. Sin embargo, es importante insistir en que de esto no se deriva un estancamiento en la visión nostálgica de la traducción como misión imposible. Muy al contrario, como han percibido algunos traductólogos, si hay algo que la deconstrucción puede aportar a su campo es justamente la liberación definitiva de la esclavitud a la noción de equivalencia que había marcado la tradición anterior. $^{4}$

\footnotetext{
${ }^{2}$ Si bien, el dominador tampoco posee la lengua que llama suya: "parce que la langue n'est pas son bien naturel, par cela même il peut historiquement, à travers le viol d'une usurpations culturelle, c'est-à-dire toujours d'essence coloniale, feindre de se l'approprier pour l'imposer comme 'la sienne'”' (45).

${ }^{3}$ En G. Deleuze (Différence et répétition) encontramos una elaboración original de esta idea. El sentido es visto por él como "génesis de lo verdadero" y la verdad es, en consecuencia, "resultado empírico del sentido," es decir, cuestión de producción y no de adecuación (256).

${ }^{4}$ Citamos a Gentzler: "While not offering a specific 'translation theory' of its own, deconstruction, however, does 'use' translation often both to raise questions regarding the nature of language and 'being-in-language' as well as to suggest that in the process of translating texts, one can come as close as is possible to that elusive notion of experience of différance, which 'underlies' their approach. Such thinking about the nature of translation and the nature of language, thus, becomes important to translation theorists, not because it necessarily defines another approach, but because it deepens and broadens the conceptual framework by which we define the very field itself. I suggest that the shift to a more philosophic stance from which the entire problematic of translation can be better viewed may not only be beneficial for translation theory, but that after such a confrontation, the discourse which has limited the development of translation theory will invariably undergo a transformation, allowing new insights and fresh interdisciplinary approaches, breaking, if you will, a logjam of stagnated terms and notions" (145).
} 
El relato autobiográfico de Derrida tiene implicaciones que van más allá de una crítica concreta a la política francesa en Argelia, lo que le interesa destacar es el carácter originariamente colonial de toda cultura: "Toute culture est originairement coloniale. . . . Toute culture s'institue par l'imposition unilatérale de quelque 'politique' de la langue" (Le monolinguisme 68).

La lengua es uno de los instrumentos más poderosos en este proceso uniformador, puesto que es a través del acto de nombrar lo diferente que se sanciona su apropiación y se redefine su valor. Esta problemática está estrechamente ligada a la cuestión de la traducción, y tiene consecuencias concretas para la corriente actual de descubrimiento y recuperación para occidente de las literaturas de las ex colonias-Lepenies prefiere el término "periferia." En este contexto, el papel de la traducción para dar acceso a obras que muy pocos podrán leer en su idioma original resulta esencial y sobre el traductor recae una enorme responsabilidad. Uno de los mayores riesgos que se corre al traducir estas obras es precisamente la caída en ese efecto de uniformización al que nos referíamos. Laurence Venuti ha sido una de las voces más enérgicas en la denuncia de esta "domesticación" del texto de partida al emplear en su traducción una especie de translatese que tiende a limar diferencias en lugar de darles libre juego en la lengua de llegada. Güneli Gün, una traductora turcoamericana cuya traducción del turco fue criticada en el Times Literary Supplement por su excesivo idiomatismo norteamericano, se defiende contundentemente en la misma publicación:

When I first read the great Russian writers in English, I was amazed that Tolstoy, Dostoevsky and Gogol sounded exactly alike. Not only that, the Germans sounded like the Russians, who in turn sounded like the great writers of Spanish, Italian and French literatures. What the British canon considers transparency isn't transparent at all, but is mediated; where the British see transparency, I see an inflated Matthew Arnold, subsuming authors from various cultures into the dominant voice that defined the British Empire, thereby "domesticating" the foreign element. (14)

En la misma línea, Gayatri Spivak lamenta la penosa situación ante la que nos encontramos cuando la traducción produce la ficción de hacer la prosa de una mujer palestina idéntica en su forma y estilo a la de un hombre de Taiwan (Spivak 69). En este sentido resulta esclarecedora la crítica que hace Philip E. Lewis a la versión inglesa de "La mythologie blanche." Lewis nos muestra cómo, aun manteniendo una aparente fidelidad literal, la nueva traducción neutraliza los efectos rompedores del texto en francés aplacando su carácter subversivo. Ante este peligro de domesticación, Lewis aboga por lo que él llama la "traducción abusiva," es decir, una traducción que no enmascare los puntos discordantes, y que parta de una exigencia de fidelidad mucho más allá de la sustancia semántica para abarcar también las modalidades de la expresión y las estrategias retóricas (38ss.).

\footnotetext{
${ }^{5}$ Esto podrá achacarse algunas veces a la mala calidad de la traducción, pero no siempre. En muchos casos la dificultad de trasladar la peculiaridad del estilo entre lenguas tan dispares reside en el hecho de que los procedimientos retóricos innovadores en la lengua de partida no tienen paralelo en la de llegada. Otras veces, se trata simplemente de que lo que en una lengua es rompedor, puede parecer en la otra reaccionario, y a la inversa. De ahí que resulte extremadamente difícil dar una idea adecuada de los logros de las literaturas lejanas, y que a simple vista y juzgando a partir de traducciones, casi siempre parezcan mejores los autores que no necesitan ser traducidos al inglés por ser esta ya su lengua de escritura.
} 
Es muy interesante observar la situación actual del inglés, que está siendo literalmente "bombardeado" — en el mejor sentido-y acrecentado por la obra pujante de autores que escriben en inglés teniendo como lengua materna otra distinta; ${ }^{6}$ es el fenómeno condensado en el lema The Empire Writes Back. La traducción de la lengua del otro se hace innecesaria cuando éste se apropia de la nuestra como suya. Sin embargo, esta adopción de la lengua del centro por parte de la periferia supone también en cierta medida una claudicación. El indio que desea hacer oír su voz anticolonialista sabe que ha de hacerlo en inglés y no en hindi si quiere ser escuchado. Su elección de la lengua de la antigua metrópoli, en lugar de servirle como herramienta de emancipación, confirma el éxito de la asimilación cultural fruto de la etapa colonial. Esta paradoja la plantea lúcidamente Derrida en "Le langage et les institutions philosophiques," incluido en Du droit à la philosophie:

Essayez d'expliquer à quelqu'un qui détient à la fois la force et la force de loi que vous voulez conserver votre langue. Il faudra que vous appreniez la sienne pour le convaincre. Une fois que, par souci de persuasion rhétorique ou politique, vous vous êtes approprié la langue du pouvoir, que vous la maîtrisez assez pour tenter de convaincre ou de vaincre, vous êtes d'avance vaincu à votre tour, et convaincu d'avoir tort. L'autre, le roi, a démontré par le fait de la traduction qu'il avait raison de parler sa langue et de vous l'imposer. En lui parlant dans sa langue, vous reconnaissez sa loi et son autorité, vous lui donnez raison, vous contresignez l'acte qui lui donne raison de vous. (299)

$\mathrm{Al}$ percatarse de este efecto opuesto al deseado, no pocos escritores de la periferia han retornado a su idioma materno, aun a riesgo de perder gran número de lectores. Evidentemente, en estos casos la decisión de escribir en una u otra lengua no es estrictamente-y ni siquiera en primer lugar- "literaria," sino política ${ }^{7}$.

Vemos que la coyuntura internacional nos coloca ante un problema doble y paradójico: por un lado, la convivencia mundial hace necesario potenciar el contacto entre las culturas, el mutuo entendimiento; por otro lado, el objetivo parece estar más bien en preservar las diferencias para evitar su neutralización. Pero en opinión de Lepenies el motivo de fondo que se esconde tras la potenciación de esta última actitud por parte de los países ricos no está exento de mala fe. El deseo de mantener la diferencia viene determinado por una mirada eurocéntrica o americocéntrica, desde una posición de descongestión y de saturación. Las culturas "hambrientas," sin embargo, aspiran generalmente a la indiferenciación y la asimilación, porque esperan que de la imitación del estilo de vida "occidental" resultará una equiparación de las oportunidades de vida. Quien desee salvar la diversidad de modos de vida, deberá trabajar por la equiparación de las oportunidades (11).

\footnotetext{
${ }^{6}$ Nótese que el premio más renombrado de las letras inglesas, el Booker Prize for Fiction, fue otorgado en 1981 a Salman Rushdie; en los doce años que siguieron lo obtuvieron dos australianos, un maorí, un sudafricano, una escritora de origen polaco, un nigeriano y un japonés. Lepenies comenta con cierta ironía que esta es ahora la rutina, y que sólo cuando un inglés "de verdad" recibe el premio la gente aguza los oídos.

${ }^{7}$ Encontramos también casos como el de Cioran, que adoptó el francés y se negó a escribir en rumano como gesto de protesta por la falta de libertades en su país. En el otro extremo del espectro se encuentran posturas absurdas, como los esfuerzos de reafricanización de los americanos negros en Nueva York mediante la enseñanza a los niños del kisuaheli, una lengua que originariamente no era hablada por ninguno de los esclavos que llegaron al nuevo mundo (Lepenies 105).
} 
Parece pues que si queremos preservar la diferencia de un modo democrático, hacerla prosperar en la lengua de llegada, vamos a tener que replantearnos la noción de fidelidad que se ha venido manejando en traducción. Barbara Johnson se refiere con ironía a la crisis de la fidelidad que se observa en nuestros días tanto en la traducción como en el matrimonio y propone la bigamia como alternativa:

... the crisis in marriage and the crisis in translation are identical. For while both translators and spouses were once bound by contracts to love, honor, and obey, and while both inevitably betray, the current questioning of the possibility and desirability of conscious mastery makes that contract seem deluded and exploitative from the start. But what are the alternatives? Is it possible simply to renounce the meaning of promises or the promise of meaning? Fortunately, I must address translation, not matrimony. Yet the analogy between the two is extremely far-reaching. It might, however, seem that the translator ought, despite or perhaps because of his or her oath of fidelity, to be considered not as a duteous spouse but as a faithful bigamist, with loyalties split between a native language and a foreign tongue. Each must accommodate the requirements of the other without their ever having the possibility to meet. The bigamist is thus necessarily doubly unfaithful, but in such a way that he or she must push to its utmost limit the very capacity for faithfulness. (142-143)

Pero la clave definitiva para resituarnos desde el punto de vista ético-adoptado tradicionalmente - al erótico nos la va a dar la noción de supervivencia, que Derrida toma de Benjamin (Überleben) situándola en el centro de su reflexión traductológica. Como veíamos más arriba, en el movimiento de la traducción entra en juego un deseo bidireccional típico del erotismo: deseo de atraer hacia sí al objeto de esa pasión, deseo de poseerlo, de fagocitarlo y asimilarlo al propio cuerpo, pero también deseo de identificarse con el otro, de asimilarse a él y de diseminar por completo el propio yo en la otredad. La afirmación amorosa se confunde con la pulsión de aniquilación del objeto amado. Para hallar el lazo de unión entre erotismo, traducción y supervivencia habremos de recordar una concisa frase de Bataille: "El erotismo es la aprobación de la vida hasta en la muerte" (15). Esta aparente paradoja nos remite directamente a la relación establecida por Derrida en textos como "Des Tours de Babel," Living On: Border Lines, o L'oreille de l'autre entre traducción y supervivencia. Derrida, evocando a Benjamin, define la tarea del traductor como la necesidad de responder a la exigencia de supervivencia que se halla inscrita en la estructura misma del original (L'oreille 162). Con esto Derrida quiere subrayar que la comprensión profunda de un texto como texto original no se alcanza si no se tiene en cuenta su "estructura superviviente." Esta perspectiva permite un replanteamiento de la antiquísima discusión acerca de la fidelidad al original. Al hilo de lo dicho, el único respeto, la única fidelidad posible por parte del traductor es a este designio constitutivo del original: el de sobrevivir. La supervivencia de un texto sólo puede garantizarla el double bind de la traducción: muerte y vida, traducibilidad e intraducibilidad al mismo tiempo. En palabras de Derrida:

Übersetzung and "translation" overcome, equivocally, in the course of an equivocal combat, the loss of an object. A text lives only if it lives on [sur-vit], and it lives on only if it is at once translatable and untranslatable. . . . Totally translatable, it disappears as a text, as writing, as a body of language [langue]. Totally untranslatable, even within what is believed to be one language, it dies immediately. Thus triumphant translation is neither the life nor the death of the text, only or already its living on, its after life, its life after death. ("Living On" 102-3) 
El movimiento traductor pone pues en práctica la tensión contradictoria de amor y destrucción, que nos ha servido de punto de partida para desplazarnos desde una lectura eticopolítica hacia una erótica de la traducción. Para Gayatri Spivak, la tarea de la traducción tiene mucho que ver con la entrega a la retórica del original, y esta entrega es "más de naturaleza erótica que ética" (71). A la hora de traducir, la "fidelidad" deberá ser entendida no como una imposición castrante, sino como un deseo activo de acrecentar, de colmar amorosamente al original. También Derrida ve en la traducción un "movimiento de amor" (223). Personalmente, no se me ocurre otro modo más intenso de vivir el cuerpo a cuerpo con un texto que traducirlo.

\section{OBRAS CITADAS}

Bassnett, Susan, and Harish Trivedi, eds. Post-colonial translation. London: Routledge, 1999.

Bataille, Georges. El erotismo. [1957] Barcelona: Tusquets. 1997

Benjamin, Walter. Introd. "Die Aufgabe des Übersetzers." En Tableaux parisiens de Baudelaire. Heidelberg: Suhrkamp. 1923.

De Man, Paul. Resistance to Theory. Minneapolis: U of Minnesota P, 1986.

Derrida, Jacques. Donner le temps I. La fausse monnaie. Paris: Galilée, 1990.

_. Du droit à la philosophie. Paris: Galilée, 1990.

"Living on: Border Lines," Deconstruction and Criticism. Bloom, et al. New York: The Seabury Press, 1979. 1996.

Le monolinguisme de l'autre ou la prothèse de l'origine. Paris: Galilée,

L'oreille de l'autre. Autobiographies, transferts, traductions. [Textes et débats avec Jacques Derrida, sous la direction de Claude Levesque et. Christie V.

McDonald.] Montréal: vlb, 1982.

- Memoirs pour Paul de Man. Paris: Galilée, 1988.

—. Psyché. Inventions de l'autre. Paris: Galilée, 1987.

_. Schibboleth. Pour Paul Celan. Paris: Galilée, 1984.

—. Suplementos Anthropos 15: Jacques Derrida - “Cómo no hablar?” y otros textos. Marzo, 1989.

Gentzler, Edwin. Contemporary Translation Theories. Routledge, 1993.

Graham, J.F., ed. Difference in Translation. Ithaca: Cornell UP, 1985.

Gün, Güneli "Something wrong with the language." Times Literary Supplement. 12 March, 1999. 
Hirsch, Alfred. Der Dialog der Sprachen. Studien zum Sprach-und Übersetzungsdenken Walter Benjamins und Jacques Derridas. München: Wilhelm Fink, 1995. ed. Übersetzung und Dekonstruktion. Frankfurt: Suhrkamp, 1997.

Lepenies, Wolf (1997): "Die Übersetzbarkeit der Kulturen." Die Sprache der anderen. Ed. Anselm Haverkamp. Frankfurt: Fischer, 1997.

Spivak, Gayatri. "Die Politik der Übersetzung." Die Sprache der anderen. Ed. Anselm Haverkamp. Frankfurt: Fischer, 1997.

Venuti, Lawrence. The Translator's Invisibility: A History of Translation. London: Routledge, 1995.

The Scandals of Translation. London: Routledge, 1998.

Wetzel, Michael und Jean-Michel Rabaté, eds. Ethik der Gabe. Denken nach Jacques Derrida. Berlin: die blaue Eule, 1993.

Wills, David "Gespaltene Zunge. Eine Antwort auf Jacques Derrida." Die Sprache der anderen. Ed. Anselm Haverkamp. Frankfurt: Fischer, 1997. 